\title{
RV Tau Variables in Globular Clusters: Clues on Their Nature?
}

\author{
Stephen C. Russell \\ Department of Physics and Mathematical Physics, \\ University of Adelaide, Adelaide, SA 5005, Australia \\ srussell@physics.adelaide.edu.au \\ Received 1997 October 21, accepted 1998 March 19
}

\begin{abstract}
We report on the detailed elemental abundances of RV Tau variables that reside in globular clusters, and how they compare with those of RV Tau variables in the field. Surprisingly, they seem to show definite differences, and at this stage, it is not clear why this is so.
\end{abstract}

Keywords: stars: AGB and post-AGB — stars: evolution — stars: variables

\section{Introduction}

RV Tau variables appear to be either blue-loop stars, at the very end of their evolution along the asymptotic giant branch (AGB), or are already post-AGB stars evolving bluewards across the instability strip (Jura 1986). Some years ago, Luck \& Bond (1989) found evidence for systematic s-process underabundances in field RV Tau variables, and stars like them. If this were true, it would have serious implications for our accepted view of AGB evolution, the nature of RV Tau variables, and the nucleosynthesis of s-process elements in the universe.

Luck \& Bond (1989) noted that the s-process abundances were reminiscent of those found in extreme halo red giants. Yet RV Tau variables have much higher metallicities than extreme halo stars. Luck and Bond suggested that either:

1. The stars reflect their original s-process deficiencies relative to $\mathrm{Fe}$, but severe mass-loss has reduced the hydrogen abundance in the atmosphere, thereby raising the $[\mathrm{Fe} / \mathrm{H}]$ ratios* to the observed levels.

2. The stars have normal s-process abundances, but because of their low 2nd ionisation potentials, these elements are selectively over-ionised by Lyman-continuum photons. The key to this possibility is the observed systematic deficiency in Sc in these stars, being the only light metal with a sufficiently low second ionisation potential.

These two alternatives could be tested by observing RV Tau variables, and stars like them, in globular clusters, and comparing them with their red giant companions. If a hydrogen deficiency caused the s-process deficiencies, RV Tau variables would show higher $[\mathrm{Fe} / \mathrm{H}]$ ratios than the normal red giants, but the

\footnotetext{
${ }^{*}[\mathrm{X} / \mathrm{H}]$ holds the usual meaning of $\log N(\mathrm{X} / \mathrm{H})_{\text {star }}-\log$ $N(\mathrm{X} / \mathrm{H})_{\odot}$
}

same [s-process/Fe] ratios. If over-ionisation were the cause, RV Tau variables should show the same $[\mathrm{Fe} / \mathrm{H}]$ ratios as the red giants, but lower [s-process/Fe] ratios.

In order to test these possibilities, I analysed the spectra of three RV Tau, or RV Tau-like variables, in globular clusters, and their red giant companions (Russell 1997). My conclusion was that RV Tau variables in globular clusters show almost none of the characteristics of the stars in the field, as determined by Luck \& Bond (1989, and references therein). The $[\mathrm{Fe} / \mathrm{H}]$ is quite normal, and the s-process elements show no evidence for depletion relative to iron. If anything, s-process elements, and scandium, show enhancements compared with their red giant companions.

This situation was somewhat puzzling, and initially it was thought that the chemical characteristics of RV Tau variables as a class were poorly determined due to the small numbers of objects studied so far. Fortunately, some degree of order and consensus have been arrived at through the recent work of Gonzalez, Lambert and colleagues (Gonzalez \& Wallerstein 1994; Gonzalez, Lambert \& Giridhar 1997a; Gonzalez, Lambert \& Giridhar 1997b; Gonzalez \& Lambert 1997; Giridhar, Rao \& Lambert 1994).

The intention of this paper is to combine the results of the different chemical abundance studies of RV Tau variables, to see if we can throw any new light on the final stages in the evolution of stars similar to our own Sun, and the nucleosynthetic processes that occur.

\section{Results}

\subsection{Globular Cluster Stars}

The mean of results from Russell (1997) for the stars V11 in M2 and SH11 in M13; from Gonzalez \& Lambert (1997) for stars V11 in M2, V84 in M5, 
Table 1. Adopted abundances

\begin{tabular}{|c|c|c|c|c|c|c|c|}
\hline Element & $A$ & $\begin{array}{l}\text { Solar } \\
{[\mathrm{M} / \mathrm{H}]}\end{array}$ & $\begin{array}{l}\text { Globular } \\
{[\mathrm{M} / \mathrm{Fe}]}\end{array}$ & $\sigma$ & $\begin{array}{l}\text { Field } 1 \\
{[\mathrm{M} / \mathrm{Fe}]}\end{array}$ & $\sigma$ & $\begin{array}{l}\text { Field } 2 \\
{[\mathrm{M} / \mathrm{Fe}]}\end{array}$ \\
\hline $\mathrm{Na}$ & 11 & $6 \cdot 33$ & $0 \cdot 46$ & $0 \cdot 13$ & 0.99 & $0 \cdot 26$ & 0.55 \\
\hline $\mathrm{Mg}$ & 12 & $7 \cdot 58$ & $0 \cdot 32$ & $0 \cdot 10$ & $0 \cdot 14$ & $0 \cdot 10$ & $0 \cdot 49$ \\
\hline $\mathrm{Al}$ & 13 & $6 \cdot 47$ & $0 \cdot 54$ & $0 \cdot 35$ & $0 \cdot 98$ & - & 0.53 \\
\hline $\mathrm{Si}$ & 14 & $7 \cdot 55$ & $0 \cdot 47$ & $0 \cdot 06$ & $0 \cdot 71$ & $0 \cdot 09$ & $0 \cdot 28$ \\
\hline $\mathrm{S}$ & 16 & $7 \cdot 38$ & $0 \cdot 88$ & $\ldots$ & $1 \cdot 29$ & $0 \cdot 23$ & $\ldots$ \\
\hline $\mathrm{Ca}$ & 20 & $6 \cdot 36$ & $0 \cdot 35$ & $0 \cdot 08$ & $-0 \cdot 21$ & $0 \cdot 14$ & $0 \cdot 00$ \\
\hline $\mathrm{Sc}$ & 21 & $3 \cdot 10$ & $0 \cdot 06$ & $0 \cdot 07$ & $-0 \cdot 99$ & $0 \cdot 12$ & $-0 \cdot 49$ \\
\hline $\mathrm{Ti}$ & 22 & $4 \cdot 99$ & $0 \cdot 26$ & $0 \cdot 01$ & $-0 \cdot 58$ & $0 \cdot 19$ & $0 \cdot 09$ \\
\hline V & 23 & $4 \cdot 00$ & $0 \cdot 35$ & $0 \cdot 39$ & $\ldots$ & 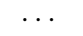 & $0 \cdot 01$ \\
\hline $\mathrm{Cr}$ & 24 & $5 \cdot 67$ & $0 \cdot 08$ & $0 \cdot 14$ & $0 \cdot 08$ & $0 \cdot 05$ & $-0 \cdot 10$ \\
\hline $\mathrm{Mn}$ & 25 & $5 \cdot 39$ & $-0 \cdot 36$ & $0 \cdot 11$ & $0 \cdot 19$ & $0 \cdot 20$ & $-0 \cdot 28$ \\
\hline $\mathrm{Co}$ & 27 & $4 \cdot 92$ & $0 \cdot 08$ & $0 \cdot 60$ & $0 \cdot 18$ & $0 \cdot 06$ & $0 \cdot 07$ \\
\hline $\mathrm{Ni}$ & 28 & $6 \cdot 25$ & $0 \cdot 10$ & $0 \cdot 05$ & $0 \cdot 04$ & $0 \cdot 08$ & $0 \cdot 08$ \\
\hline $\mathrm{Cu}$ & 29 & $4 \cdot 21$ & $-0 \cdot 29$ & $\ldots$ & $\ldots$ & $\ldots$ & $-0 \cdot 29$ \\
\hline $\mathrm{Zn}$ & 30 & $4 \cdot 60$ & $0 \cdot 13$ & $0 \cdot 12$ & $1 \cdot 09$ & $0 \cdot 27$ & $-0 \cdot 04$ \\
\hline $\mathrm{Y}$ & 39 & $2 \cdot 24$ & $-0 \cdot 06$ & $0 \cdot 06$ & $-0 \cdot 51$ & $0 \cdot 24$ & $-0 \cdot 72$ \\
\hline $\mathrm{Zr}$ & 40 & $2 \cdot 60$ & $0 \cdot 51$ & $0 \cdot 17$ & $\ldots$ & $\ldots$ & $-0 \cdot 83$ \\
\hline $\mathrm{Ru}$ & 44 & $1 \cdot 84$ & $0 \cdot 14$ & $\ldots$ & $\ldots$ & $\ldots$ & $\ldots$ \\
\hline $\mathrm{Ba}$ & 56 & $2 \cdot 13$ & $0 \cdot 41$ & $0 \cdot 10$ & $-0 \cdot 20$ & $\ldots$ & $-0 \cdot 38$ \\
\hline La & 57 & $1 \cdot 22$ & $0 \cdot 09$ & $0 \cdot 31$ & $\ldots$ & $\ldots$ & -0.55 \\
\hline $\mathrm{Ce}$ & 58 & 1.55 & $0 \cdot 10$ & $0 \cdot 15$ & $0 \cdot 69$ & $\ldots$ & -0.53 \\
\hline $\operatorname{Pr}$ & 59 & $0 \cdot 71$ & $0 \cdot 13$ & $\ldots$ & $\ldots$ & $\ldots$ & $-1 \cdot 77$ \\
\hline $\mathrm{Nd}$ & 60 & $1 \cdot 50$ & $0 \cdot 11$ & $0 \cdot 17$ & $\ldots$ & $\ldots$ & $-0 \cdot 66$ \\
\hline $\mathrm{Sm}$ & 62 & $1 \cdot 00$ & $0 \cdot 38$ & $0 \cdot 02$ & $0 \cdot 52$ & $\ldots$ & $-0 \cdot 61$ \\
\hline $\mathrm{Eu}$ & 63 & 0.51 & $0 \cdot 47$ & $0 \cdot 11$ & $0 \cdot 04$ & $\ldots$ & $-0 \cdot 05$ \\
\hline Dy & 66 & $1 \cdot 10$ & $0 \cdot 46$ & $\ldots$ & $\ldots$ & $\ldots$ & $\ldots$ \\
\hline
\end{tabular}

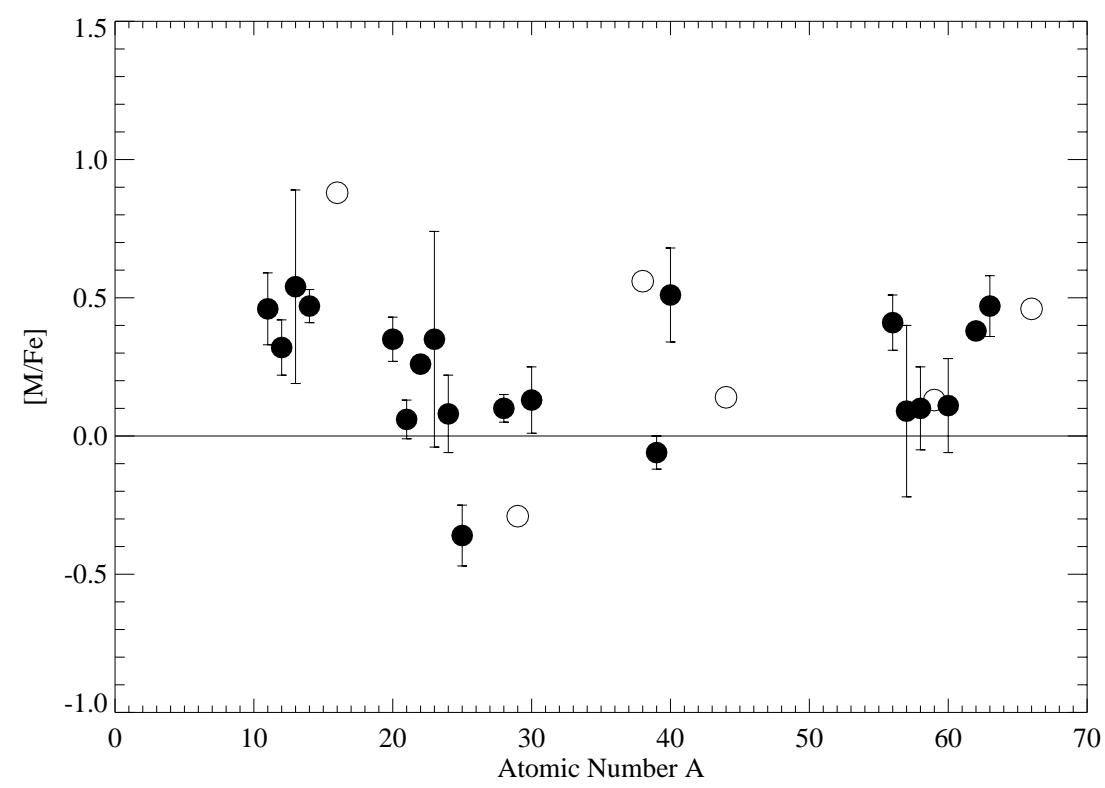

Figure 1-Average $[\mathrm{M} / \mathrm{Fe}]$ ratios for RV Tau variables in globular clusters.

V17 in M28, and V2 in M10; and from Gonzalez \& Wallerstein (1994) for V1 in $\omega$ Cen, are presented in column 4 of Table 1. For V11 in M2, the weighted mean of the results from the two references was used. The star V6 in M56 has been discarded since the abundances are discrepant. The solar abundances adopted in Russell (1997) were used in each case, except for V1 in $\omega$ Cen, where a differential solar analysis was used.
The results are also shown in Figure 1, where the filled circles with error bars had more than one measure for the abundance, and the open circles had just one measure. The error bars are the standard deviations in the mean of the abundances derived from all the stars.

The first result is that the $[\mathrm{Fe} / \mathrm{H}]$ abundances for the RV Tau variables, compared with the $[\mathrm{Fe} / \mathrm{H}]$ abundances of the red giants in the same clusters, 


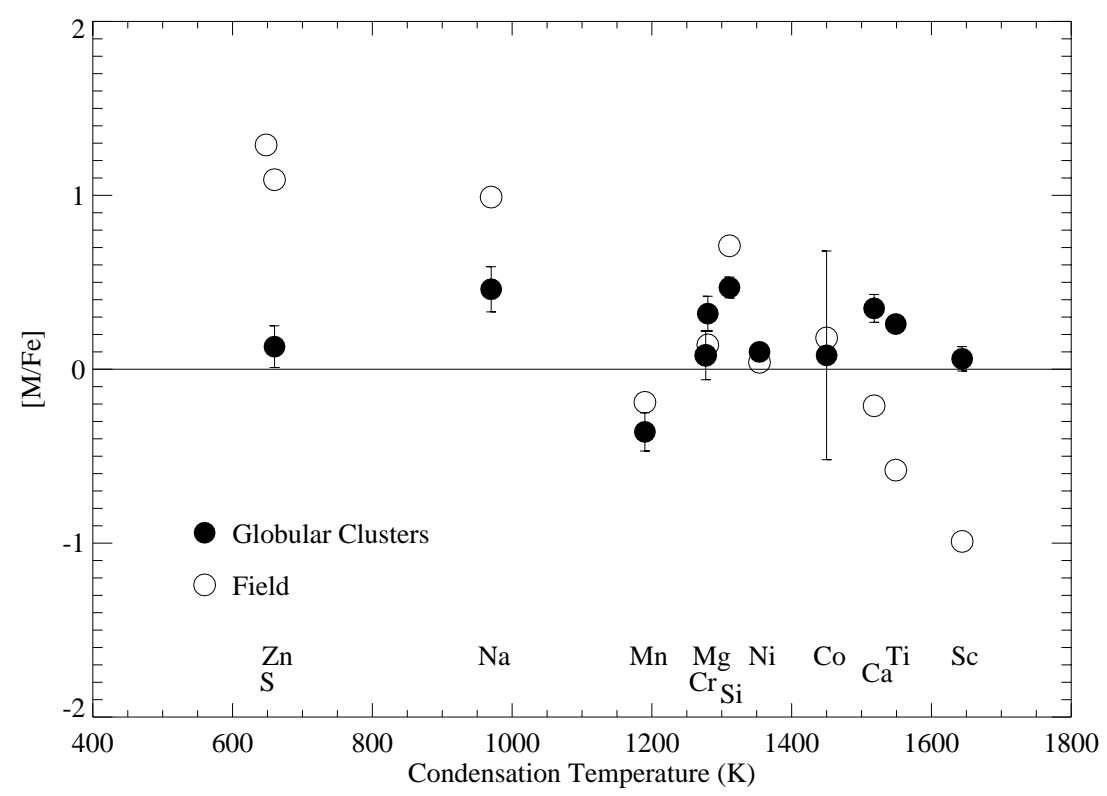

Figure 2-Average $[\mathrm{M} / \mathrm{Fe}]$ for RV Tau variables in the field and in globular clusters, against condensation temperature.

are $-0 \cdot 07 \pm 0 \cdot 04$. This value was calculated without including V2 in M10, as this star was felt to be discrepant by Gonzalez \& Lambert (1977). This shows that there are negligible differences between the RV Tau variable iron abundances and those of the local environs. Certainly there is no suggestion of an overabundance of iron. This means that it is unlikely that these variables suffer from a hydrogen deficiency.

There is also no sign of an underabundance in s-process elements relative to iron. Figure 1 shows clearly that there is actually an overabundance of s-process elements, relative to the Sun. This is likely to be true also of the variable stars compared to their local environs, as shown by two of the stars studied by Russell (1997). Based on these results, after correcting for the detailed differences between the companion red giants and the Sun, all s-process elements, including $\mathrm{Y}$, would lie above the zero line.

Although the results are provocative, it is hard to say how significant they are at this stage. Barium is the most significantly overabundant sprocess element, and it depends on two or three saturated resonance lines. Further work on refining the measurements in the future may hold the key to determining the exact processes involved in production of the s-process elements.

The second major result from this work is the significant overabundance of the light elements $\mathrm{Na}$, $\mathrm{Mg}, \mathrm{Al}$ and Si. These data are on a firmer footing than those for the s-process elements, since they have been observed in more stars, and Si, particularly, depends on many more lines.

The Na excess has already been discussed in Gonzalez \& Lambert (1997), with regard to V84 in M5, and the possibility that it is a product of ON-cycling (Langer, Hoffmann \& Sneden 1993). The present work demonstrates that the Na excess is common to the RV Tau variables in globular clusters. What is more, the $\mathrm{Al}$ excess suspected by Gonzalez \& Lambert (1997) was demonstrated by Russell (1997) to be a true excess in two RV Tau type variables, compared with their companion red giants. This more firmly supports the ON-cycling explanation for light element excesses.

$\mathrm{Mg}$ and $\mathrm{Si}$ are both $\alpha$-elements, and the work of Russell (1997) illustrates that the over abundances in these elements, relative to the Sun, are not significantly different from the abundances observed in the companion red giants. This is in agreement with the $\omega$ Cen RV Tau variable V1 (Gonzalez \& Wallerstein 1994), though in apparent disagreement with the star V84 in M5 (Gonzalez \& Lambert 1997). The statistics are too poor to make firm conclusions here, but present indications are that the $\alpha$-elements in RV Tau variables in globular clusters are usually in normal abundance ratio to Fe, compared with neighbouring red giants.

\subsection{Field Stars}

The full results for the average abundances for field RV Tau variable stars are shown in Table 1. Columns 6 and 7 ('Field 1' and ' $\sigma$ ' respectively) give the average abundances and standard deviations from the work of Gonzalez, Lambert \& Giridhar (1997a); Gonzalez, Lambert \& Giridhar (1997b); and Giridhar, Rao \& Lambert (1994). Column 8 ('Field 2') gives the results from Luck \& Bond (1989, and references therein), for comparison purposes. 


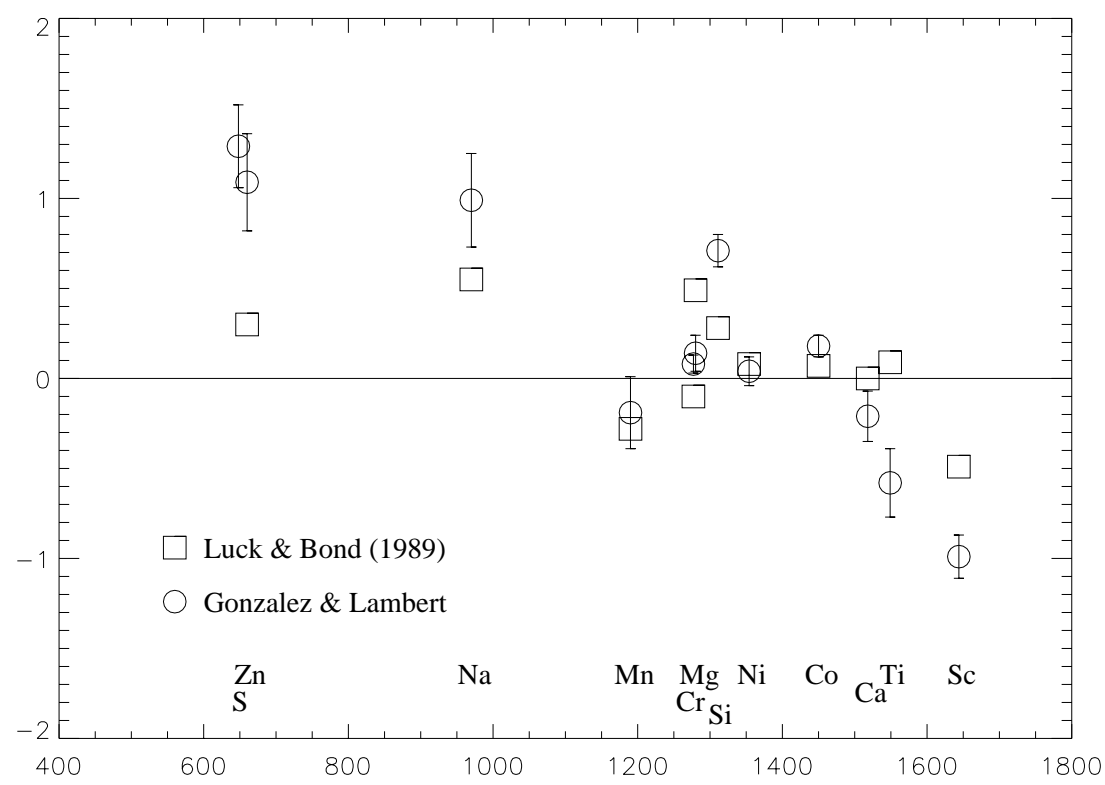

Figure 3-Abundance distribution results of Luck \& Bond (1989) compared those of Gonzalez, Lambert and coworkers.

It is becoming increasingly apparent that the detailed chemical abundance distribution of field RV Tau variables is best explained if we plot the abundances against the condensation temperatures for those elements. In Figure 2 the $[\mathrm{M} / \mathrm{Fe}]$ abundance ratios for the 'Field 1' field stars (column 6 of Table 1) are plotted, as open circles, against the condensation temperatures. For comparison, the mean abundances of the globular cluster stars (column 4 of Table 1) are plotted as filled circles, with the appropriate error bars. This clearly demonstrates the difference between the two sets of stars. The field stars track the abundance distribution of the interstellar medium (see, for instance, Gonzalez, Lambert \& Giridhar 1997), while the globular cluster stars show no such trend.

The only problem is that the work of Luck \& Bond (1989) seems to disagree with these results. Figure 3 compares the results of Luck \& Bond with the more recent results given in column 6 of Table 1 . Clearly, it is the critical elements S, Zn, Na, Sc, and $\mathrm{Ti}$ that will decide the issue. These are the very same elements that will decide the differences and similarities between field and globular cluster stars. On the grounds of condensation temperature alone, therefore, there is still a case to be answered. There is no overlap in objects between the field stars studied in 'Field 1' and those in 'Field 2'. Work has to be done to repeat observations and analyses of the stars studied by Luck \& Bond, and those in the references quoted in that paper.

Further clues can be obtained by asking how the abundances of the s-process elements match between the Gonzalez and Luck \& Bond studies. The Luck \& Bond study shows uniformly negative relative abundances to iron, for all s-process elements. Un- fortunately, the Gonzalez study did not concentrate on s-process elements, and only returned significant results for $\mathrm{Ba}$, and these were consistent with those from the Luck \& Bond study. Again, it would be profitable both to carry out a new study of the Luck \& Bond stars, and re-observe the Gonzalez study stars to determine the s-process elements.

\subsection{Difference Between Field and Globular Cluster Stars}

No matter what assumptions we make about the field RV Tau variables, it still seems that the globular cluster RV Tau variables are different. If the results of 'Field 2' are to be believed, we must explain why cluster variables do not show s-process element under abundances. No explanations for this difference have been published in the literature. Presumably one of the options suggested by Luck \& Bond (1989) operates in field variables, but not in cluster variables. Since the 'over-ionisation' option should reasonably be expected to operate in both field and globular variables, this seems the least likely explanation. At this stage, however, it seems inappropriate to pursue the explanation in detail until the results have been confirmed.

If the results of 'Field 1' are to be believed, then three options are offered by Gonzalez \& Lambert (1997) to explain the differences. The explanations depend on the assumption that field variables undergo dust/gas separation, and cluster variables do not. Though the photospheric metallicities of field stars range through the metallicities observed in globular clusters, they are supposed to have achieved these low metallicities through selective loss of dust relative to gas. Therefore, either the higher intrinsic metallicity of the stars, or a higher evolutionary mass, might be 
responsible for the more intense, or longer duration, stellar wind.

A third possibility is that the environment in a globular cluster is responsible for the difference, by precluding binary formation. There is a possibility that binary stars are responsible for the field RV Tau abundance patterns, as they are for the peculiar abundance patterns of Ba-stars. Evidence that binaries may be rare in globular clusters comes from Mayor, Duquennoy \& Udry (1996), who reported that in $\omega$ Cen there are only about $20 \%$ as many binary systems as are found in the field.

Again, there is still a great deal of work to be done to establish the facts of the case, before too much effort is directed towards explaining the apparent systematic effects.

\section{Conclusions}

It seems that RV Tau variable stars, and stars like them, in globular clusters, show significant overabundances in both s-process elements and the light elements $\mathrm{Na}$ and $\mathrm{Al}$. Not only is this true in comparison with the Sun, but also compared with neighbouring red giant stars. However, there is no sign of the correlation between abundance and condensation temperature that is seen in field variables, at least for 'Field 1' results.

To confuse matters, the results from 'Field 2' do not agree with those from 'Field 1'. It is possible that the RV Tau class of variable star harbours a disparate set of variable types, which may account for the differences observed between the studies. It is important, now, to re-study the stars in 'Field 1 ' to determine the s-process element abundances more precisely, and to re-study the 'Field 2' stars in the same manner as the 'Field 1' stars, to see if there really is a difference between the two sets of results.

\section{Acknowledgments}

My sincere thanks to the University of Adelaide, Department of Physics and Mathematical Physics, for providing me with the facilities necessary for writing up this paper.

\section{References}

Giridhar, S., Rao, N. K., \& Lambert, D. L. 1994, ApJ, 437, 476

Gonzalez, G., \& Lambert, D. L. 1997, AJ, 114, 341

Gonzalez, G., Lambert, D. L., \& Giridhar, S. 1997a, ApJ, 479,427

Gonzalez, G., Lambert, D. L., \& Giridhar, S. 1997b, ApJ, 481,452

Gonzalez, G., \& Wallerstein, G. 1994, AJ, 108, 1325

Jura, M. 1986, ApJ, 309, 732

Langer, G. E., Hoffman, R., \& Sneden, C. 1993, PASP, 105, 301

Luck, R. E., \& Bond, H. E. 1989, ApJ, 342, 476

Mayor, M., Duquennoy, A., \& Udry, S. 1996, in Origins, Evolutions, and Destinies of Binary Stars in Clusters, ed. E. F. Milone \& J.-C. Mermilliod (San Francisco: ASP), p. 190

Russell, S. C. 1997, A\&A, 326, 1069 